\title{
Technologie des fromages grecs ${ }^{(1)}$
}

\author{
par \\ le Dr Georges KALATZOPOULOS \\ Assistant à la chaire de Technologie \\ à l'Ecole supérieure d'Agriculture d'Athènes (Grèce)
}

Si l'on veut étudier la technologie des fromages grecs et ceux des autres Pays de la Méditerranée orientale, il faut d'abord savoir que le climat et les conditions de la production laitière, c'est-à-dire le nombre et la grandeur des exploitations, ont joué un rôle primordial sur les méthodes de préparation des fromages.

La technologie utilisée aujourd'hui, non seulement par les fromageries primitives et temporaires, encore nombreuses, mais aussi par les grandes industries laitières, est le résultat d'un empirisme transmis de génération en génération, tout au long des siècles. C'est pourquoi il faut constater pour commencer qu'il n'existe pas de produits d'une technologie standardisée et, par conséquent, d'une composition définie.

C'est à cause de son climat sec que la production du lait n'a pu dépasser le million de tonnes par an que seulement après 1962. En 1971, la production totale était environ de $1400000 \mathrm{t}$ dont :

- lait de vache : 556509 t (39,73 p. 100), dont 67,9 p. 100 pour la consommation et 17,5 p. 100 pour la préparation des fromages,

- lait de brebis : 477770 t (34,11 p. 100), dont 80 p. 100 pour la préparation des fromages et 18 p. 100 pour le yaourt,

- lait de chèvre : 362724 t (25,9 p. 100), préparation du fromage,

- lait de bufflesse : 3605 t (0,26 p. 100), préparation du fromage.

Mais malgré la très relative production de fromage, $100000 \mathrm{t}$, dont $80000 \mathrm{t}$ à partir de lait de brebis, par rapport aux autres pays producteurs, la statistique du Centre national du commerce extérieur de France, indique que la Grèce garde la première place pour la consommation du fromage dans le monde.

Comment peut-on expliquer cela ? Pour la majorité des grecs, le fromage n'est pas, comme pour les français, un dessert, mais, une nourriture nécessaire pour réaliser l'équilibre des protéines. Souvent

(1) Conférence donnée le 10-12-1973 au Centre national de recherches zootechniques à Jouy-en-Josas. 
l'été, à la campagne, les paysans pendant leur repos de travail, mangent du fromage, du pain et des fruits.

Cette habitude reste la même que celle décrite par Homère et les autres écrivains dans l'antiquité, parlant de la vie quotidienne plusieurs siècles avant notre ère.

\section{Classification des fromages}

90 p. 100 des fromages grecs sont faits avec du lait de brebis, souvent mélangé avec un certain pourcentage de lait de chèvre. On peut distinguer trois catégories de fromages :

1) Fromages à pâte molle, avec un temps de maturation au minimum de 2 mois, une humidité variable, selon la qualité, de 52,5 p. 100 à 56 p. 100 , avec une teneur en matière grasse de 22 p. 100 à 15 p. 100 respectivement. Ces fromages composent 74 p. 100 de la production totale.

2) Fromages durs, très salés ou assez salés avec un temps de maturation de 3 mois, une humidité variant de 35 p. 100 à 38 p. 100 et un taux de matière grasse de 47 p. 100 à 32 p. 100 dans l'extrait sec.

3) Fromages de lactosérum, préparés surtout à partir de petitlait de fromages durs, faits de lait de brebis, et qui composent 6 p. 100 de la production totale, avec une humidité et temps de consommation très variables.

Comme la technologie se trouve au stade de la standardisation, je vais essayer de vous donner les méthodes de fabrication de certaines espèces les plus représentatives et en même temps les plus importantes comme on les prépare en industrie laitière et dans les fromageries temporaires.

\section{Feta}

\section{I. - FROMAGES A PATE MOLLE}

Le fromage à pâte molle le plus connu au monde, fait de lait de brebis, est le Feta. Pour les grecs, le Feta est le Camembert des français.

\section{Fabrication}

D'après la législation, la pasteurisation est obligatoire dans toutes les fromageries. Dans les grandes industries elle est faite à $71^{\circ} \mathrm{C}-72^{\circ} \mathrm{C}$ pendant $15 \mathrm{~s}$, tandis qu'en fromageries temporaires et primitives à $63^{\circ} \mathrm{C}-65^{\circ} \mathrm{C}$ pendant $30 \mathrm{mn}$. Bien entendu, après cette pasteurisation, la qualité bactériologique du lait n'est pas brillante. Une standardisation empirique est réalisée par un écrémage de 10 p. 100 environ, et, de cette manière, la matière grasse est ramenée à environ 5,5 p. $100-6$ p. 100 .

Un grand nombre de fromageries utilisent comme ferments lactiques le yaourt, qu'elles préparent elles-mêmes ou qu'elles achètent, dans un pourcentage de 0,5 p. $100-1$ p. 100. 
Les grandes usines prenant l'habitude des praticiens, ajoutent aussi comme levains le yaourt ou le Str. Lactis.

Mais pourquoi utilise-t-on le yaourt comme ferments pour un fromage à pâte molle?

Il y a deux causes différentes, la première est qu'on peut facilement préparer ou acheter le yaourt et la seconde est qu'il a un pouvoir acidifiant qui abaisse le $\mathrm{pH}$ du caillé dès le premier jour, plus que les autres ferments ne le font, ce qui ne permet pas le développement des bactéries coliformes. Ceci a été vérifié dans mon laboratoire, dans le cadre du programme de recherche sur le fromage Feta.

La quantité et la qualité de la présure est évidemment variable selon les professionnels. On utilise la présure en poudre en une quantité de 3,5-4 g pour 1001 de lait. Mais comme nous avons une consommation de viande de plus 5 millions d'agneaux et 2,7 millions de chevreaux $\mathrm{F}$ par an, les fromagers préparent une certaine quantité de présure à partir de leurs caillettes, qu'ils mélangent en un certain pourcentage avec de la présure en poudre. On a remarqué entre les deux modes de coagulation une différence dans la saveur des produits, et les consommateurs préfèrent les fromages faits avec le mélange des deux enzymes car ils sont plus piquants.

Le temps de prise est de $8-10 \mathrm{mn}$ et le temps de coagulation se situe entre $60 \mathrm{mn}$ à $65 \mathrm{mn}$. Le découpage est fait immédiatement après la coagulation, en cubes de $2 \mathrm{~cm}$ de long, et ensuite le caillé est laissé en repos $10 \mathrm{mn}$, suivi d'un lent brassage et encore d'un temps de repos de $10 \mathrm{mn}$.

On transfère, à l'aide d'une louche, le caillé dans des moules de dimensions variables (diamètre $43-45 \mathrm{~cm}$ et hauteur $23 \mathrm{~cm}$ ). Les moules sont remplis partiellement pour faciliter l'égouttage. Le caillé reste dans les moules pendant 3 à $4 \mathrm{~h}$, pendant l'époque chaude et $24 \mathrm{~h}$ l'hiver. Après ce temps là, on retourne les moules sur des tables spéciales. Le salage se fait le premier jour dans les moules après égouttage et avec du gros sel.

\section{Prématuration}

Le lendemain, les fromages de chaque moule, coupés en trois morceaux, avec un couteau spécial, sont mis dans des barils en bois mais pas définitivement. C'est le stade où l'égouttage continue à l'aide du sel qui pénètre dans le fromage à une température de $14^{\circ} \mathrm{C}-18^{\circ} \mathrm{C}$.

Les fromages restant dans ces salles pendant 7 à $10 \mathrm{j}$, subissent une prématuration.

Pendant cette période, les premiers jours, ils sont transvasés 3 à 4 fois dans les barils secs en leur ajoutant chaque fois du gros sel. Durant cette prématuration, les fromages subissent progressive- 
ment une fermentation avec augmentation valable de volume, et ensuite, brutalement, le volume diminue. En même temps, on constate la sortie d'une certaine quantité de lactosérum.

\section{Affinage}

Après 7 à $10 \mathrm{j}$ de prématuration, les fromages en morceaux sont lavés à l'eau froide, et, après égouttage, déposés dans des barils en bois, en prenant soin de ne pas laisser de vide entre les fromages. Dès que les barils sont fermés, on ajoute, par le trou, laissé à cet usage, de la saumure contenant 4-5 p. 100 de sel. Si les fromages doivent être consommés rapidement, en 30-40 j, on les transporte dans une cave d'affinage à $12^{\circ} \mathrm{C}$ pour un séjour de 1 mois et ensuite dans une cave froide à $4^{\circ} \mathrm{C}$. La maturation en cave à $12^{\circ} \mathrm{C}$ n'est pas indispensable si les fromages ne doivent pas être consommés avant 2 mois.

Le rendement est de 24 à 25 p. 100 en fromage mûr et en utilisant uniquement du lait de brebis.

Une espèce de Feta est le fromage Télémes. Ce fromage est préparé soit avec du lait de brebis, soit avec du lait de vache. Sa maturation se fait dans des boîtes en étain remplies de saumure.

\section{a) Kefalotyri}

\section{II. - LES FROMAGES DURS}

L'espèce de fromage dur la plus connue est représentée par le Kefalotyri, fait également avec du lait de brebis.

Ce type de fromage ressemble beaucoup au Pecorino d'Italie. Il contient à peu près 4 à 5 p. 100 de sel, et une grande partie de sa production est utilisée en rapé pour les pâtes. On peut distinguer sous la même appellation deux espèces :

1) L'espèce qui sera consommée comme fromage après un écrémage de 10 p. 100 de la quantité totale du lait et un salage moins fort.

2) L'espèce qui sera consommée en rapé pour les pâtes. Dans ce cas, on fait un écrémage du lait à 15-20 p. 100 et une coagulation incomplète. Du lactosérum on prépare un autre fromage dont nous parlerons ci-après.

Le grand volume de la production est le fait des fromageries artisanales et temporaires dans lesquelles la pasteurisation a lieu dans les cuves de fabrication à $63^{\circ} \mathrm{C}-65^{\circ} \mathrm{C}$ pendant $30-20 \mathrm{mn}$.

Les ferments ne sont pas utilisés et la maturation est due aux micro-organismes survivants de la pasteurisation. Ici aussi la qualité et la quantité de la présure est évidemment variable selon les professionnels. On poursuit un temps de coagulation de $35-40 \mathrm{mn}$ à une température de $34^{\circ} \mathrm{C}-36^{\circ} \mathrm{C}$. Le découpage est fait immédiatement 
après la coagulation en grains d'une grandeur comparable à ceux du maïs.

Dans le cas où on préparera ensuite du fromage à partir du lactosérum, le décaillage aura lieu, avant que la coagulation ne s'accomplisse. Le brassage commence immédiatement après le découpage et un réchauffement du caillé a lieu en $15-20 \mathrm{mn}$ jusqu'à $45^{\circ} \mathrm{C}-48^{\circ} \mathrm{C}$.

Après un repos de quelques minutes, on égalise et on presse à la main les grains sur toute la surface de la cuve. Dès que les grains sont collés, le fromager fait un rouleau avec le caillé dans le lactosérum pour obtenir finalement un cylindre. Il coupe ensuite ce cylindre en morceaux de la grandeur du moule dont les dimensions sont de $30 \mathrm{~cm}$ de diamètre et de $8-10 \mathrm{~cm}$ de hauteur. Les moules sont sans fond et le caillé repose sur des toiles où il est ensuite pressé à la main pour faire sortir le lactosérum. Les fromages sont pressés pendant $24 \mathrm{~h}$, ensuite mis dans la saumure pendant $24 \mathrm{~h}$, puis transportés à la salle d'affinage à une température de $12^{\circ} \mathrm{C}-14^{\circ} \mathrm{C}$, où ils sont stockés les premiers jours l'un sur l'autre et ensuite par quatre les uns sur les autres. Le salage continue avec du gros sel sur tous côtés tous les $2 \mathrm{j}$ et pendant les 2 premiers mois.

Le rendement est de $15-16$ p. 100 quand on utilise uniquement du lait de brebis.

\section{b) Kasseri}

Dans le groupe des fromages durs il y a une espèce portant l'appellation Kasseri. Ce type ressemble au fromage Caciocavallo fait en Italie à partir de lait de vache, mais en Grèce on le prépare avec du lait de brebis avec addition d'un certain pourcentage de lait de chèvre ou de vache.

Je n'insisterai pas sur les détails de la fabrication de ce fromage, plus ou moins connu, mais je vous donnerai les points les plus intéressants.

La coagulation est due à la présure avec une température d'emprésurage de $32^{\circ} \mathrm{C}-34^{\circ} \mathrm{C}$. Après découpage, le caillé subit un chauffage à $40^{\circ} \mathrm{C}$ et ensuite on le met dans de grandes toiles spéciales pour s'acidifier.

Il y a quelques années cette préparation était faite sous une large échelle par les bergers dans les alpages, parce qu'ils ne pouvaient pas transporter aussitôt leur lait dans le village. De cette manière, au lieu de vendre le lait, on vendait le caillé, tous les 2 ou 3 j, à la plus proche fromagerie. Dans ces fromageries permanentes on coupe le caillé en petits morceaux dans une cuve et l'on ajoute du lactosérum à une température de $35^{\circ} \mathrm{C}-40^{\circ} \mathrm{C}$, et ensuite le caillé est mis dans des toiles spéciales pour s'acidifier, pendant 10 à $24 \mathrm{~h}$, selon la température ambiante.

Quand, après différents test pratiques, le fromager juge que le caillé a été suffisamment acidifié, il le divise en grandes bandes et 
ajoute de l'eau chaude à $70^{\circ} \mathrm{C}$. Il prend alors une quantité de caillé proportionnelle à la quantité du fromage à préparer et, en fonction de la grandeur des bandes, le met dans une corbeille plongeant dans l'eau chaude. Le fromager manipule la corbeille jusqu'à ce que la pâte du fromage devienne élastique. Il la dépose ensuite sur une table pour la pétrir comme dans le cas du pain. Le caillé ainsi malaxé est mis dans les moules, sans toile, et il reste pour durcir jusqu'au lendemain, moment où on le transporte dans les caves d'affinage.

\section{c) Fromage dur de type Gruyère préparé de lait de brebis}

Je donnerai seulement les différences quant à la méthode de préparation, entre le type de fromage et le Gruyère original.

Temps de prise : $7 \mathrm{mn}$ et temps de découpage : $30 \mathrm{mn}$, à une température de $32^{\circ} \mathrm{C}-34^{\circ} \mathrm{C}$. La durée du brassage avant le feu est de 10-12 mn. Le chauffage se fait en deux temps, d'abord pendant $15 \mathrm{mn}$ à $44^{\circ} \mathrm{C}$ et ensuite à $52^{\circ} \mathrm{C}$ pendant $10 \mathrm{mn}$. Le temps de ressuyage est plus court que celui utilisé pour le Gruyère original, étant donné que le lait de brebis est plus riche, en extrait sec que le lait de vache. Bien qu'on n'ajoute pas de bactéries propioniques on a constaté leur présence, grâce à la pasteurisation insuffisante du lait. L'apparition des lenures est plus fréquente et la dégustation a démontré que le fromage est plus doux et rarement, ou presque jamais, amer.

\section{III. - FROMAGES DE LACTOSERUM}

Les fromages de lactosérum peuvent être distingués en deux catégories.

1) Les fromages faits par chauffage du lactosérum avec addition d'une petite quantité de lait de brebis ou de chèvre (4-5 p. 100) et portent le nom de Mizithra. Après égouttage et un fort salage, ils sont séchés à l'ombre, en plein air, dans un filet qui les protège des insectes et des oiseaux.

2) Les fromages faits par chauffage du lactosérum avec addition d'une quantité variable de lait de chèvre (20-25 p. 100) et qui seront consommés dès le premier jour d'égouttage, comme fromage frais, sous le nom de Manouri. Ce produit se vend à un prix assez élevé et garde la préférence des consommateurs. Voyons quelques détails sur sa préparation.

Le lactosérum recueilli d'une coagulation incomplète du lait de brebis, est filtré pour enlever les petits morceaux de caillé et ensuite chauffé à $70^{\circ} \mathrm{C}$. A cette température, on ajoute le lait et on continue le chauffage brutalement et en diminuant la vitesse d'agitation. L'art du fromager consiste à pouvoir, avec une légère agitation, faire monter à la surface la plus grande partie des protéines coagulées par le 
chauffage. Le facteur le plus important pour la réussite est l'acidité du lactosérum. On recueille le caillé avec une passoire et on le transporte sur des toiles, qui sont accrochées pour l'égoutter. Le lendemain, soit on en fait le salage, soit on le consomme comme fromage frais.

J'ai ainsi essayé de vous donner une image de la fabrication des fromages en Grèce, mais cette image concerne la technologie appliquée dans les industries en voie de développement ces dernières années.

Il ne faut pas oublier qu'il existe un grand nombre d'autres fromages préparés dans des conditions artisanales ou par les paysans eux-mêmes dans un besoin d'indépendance économique. Grâce aux soins méticuleux des fromagers, la réputation de certains fromages est arrivée jusqu'à la capitale. Il est donc nécessaire d'étudier ces fromages et d'essayer de les standardiser pour profiter d'une expérience très ancienne.

\section{S u $\mathbf{m} \mathbf{m}$ a r y}

\section{THE TECHNOLOGY OF GREEK CHEESE}

Information on the processing of typical national cheese was given. The 80 p. 100 of the produced greek cheese is made from sheep milk.

Among the different varieties Feta is a soft cheese made from sheep milk, wich is very popular in all the country. The ripening is made in barrels.

A local hard cheese is kefalotyri, the characteristic of which is the high content in salt (4-5 p. 100).

Two types of whey cheese are produced in Greece, especialy from the whey of kefalotyri cheese : Mizythra a hard whey cheese and Manouri a soft whey cheese made with addition of 20-25 p. 100 goat milk.

A Gruyere type cheese is also produced from sheep milk. 